\title{
Dihydroartemisinin ameliorates palmitate-induced apoptosis in cardiomyocytes via regulation on miR-133b/Sirt1 axis
}

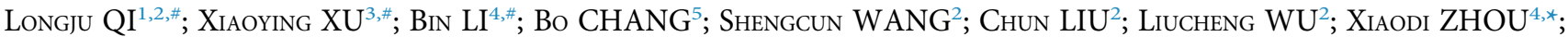 \\ QINGHUA WANG ${ }^{2, *}$ \\ 1 Affiliated Nantong Hospital 3 of Nantong University, Nantong University, Nantong, 226001, China \\ 2 Comparative Medicine Institution of Nantong University, Nantong University, Nantong, 226001, China \\ 3 Department of Gynecology and Obstetrics, Hai'an Hospital Affiliated to Nantong University, Nantong University, Nantong, 226001, China \\ 4 Department of Paediatric, Affiliated Hospital of Nantong University, Nantong University, Nantong, 226001, China \\ 5 Department of Internal Cardiovascular Medicine, the $6^{\text {th }}$ People's Hospital of Nantong City, Nantong, 226001, China
}

Key words: microRNA-133b, Palmitate acid, Cardiomyocytes, Sirt1, Dihydroaretemisinin

\begin{abstract}
Excessive fat ectopically deposited in the non-adipose tissues is considered as one of the leading causes of myopathy. The aim of this study was to investigate the role of Dihydroartemisinin (DHA) in palmitate (PAL)incubated H9c2 cells (lipotoxicity-induced cell injury model). Cell viability of PAL-treated cells was determined by MTT assay, and apoptotic regulators were examined by qRT-PCR and western blot analysis, in the absence or in the presence of DHA, respectively. Expression levels of miR-133b and Sirtl were also evaluated by qRT-PCR and western blotting examination. PAL decreased the viability of H9c2 cells and enhanced the expression of apoptotic genes. DHA reversed the effect of PAL on cell viability and lowed the level of Caspase 3 and Bax. It also lowered the expression of miR-133b, while enhanced the expression of Bcl-2. Sirtl was revealed as target of miR-133b through transcriptional regulation and the process was affected by DHA. DHA partially protected against the PAL-induced lipotoxicity by influencing the expression of miR-133b that hindered the activity of Sirt1. DHA may be used as a potential treatment in clinical management for lipotoxicity induced heart complications.
\end{abstract}

\section{Introduction}

The incidence of complications of non-communicable metabolic diseases, such as diabetes, obesity, hyperlipidemia, etc., has increased recently and became a major death cause of cardiovascular diseases (CVDs) worldwide (Heallen et al., 2019; Huntington et al., 2021). Clinical and animal model studies have shown that diabetic cardiomyopathy often presents fatty acid metabolic disorders, hyperinsulinemia and hyperglycemia (Zang et al., 2020). Lipotoxicity is defined as the exotic accumulation of excessive lipids in non-adipose tissues, resulting in cell dysfunction and apoptosis (Zhang et al., 2021a). Excessive saturated fatty acid accumulation in the heart will increase the consumption of fatty acid, and the redox reaction of fatty acid will increase by about 2 times, while the oxidative phosphorylation process of glucose will be

\footnotetext{
*Address correspondence to: Qinghua Wang, wangqh@ntu.edu.cn; Xiaodi Zhou, 13962997058@163.com

${ }^{\#}$ These authors contributed equally to this work

Received: 23 June 2021; Accepted: 28 August 2021
}

inhibited by $30-40 \%$, which will aggravate the local energy supply and oxygen consumption of the heart, resulting in local hypoxia (Kakimoto et al., 2021; Kanamori et al., 2021). Lipotoxic metabolites and reactive oxygen species lead to cardiomyocytes damage and variations of subcellular structure, such as Golgi, mitochondria and DNA, which result in the cardiomyocyte apoptosis (Liu et al., 2020). Cardiomyocyte apoptosis has been widely reported as one of the main causes of cardiac dysfunction in clinical and basic research, such as myocardial infarction, heart failure and heart ischemia-reperfusion (Noronha-Dutra and Steen, 1982; Terman and Brunk, 1998). However, at present, no appropriate prevention and treatment methods have been found, and the specific molecular mechanism is also controversial, which is worthy of further study.

Artemisinin (Art), as a sesquiterpene lactone with peroxyl group extracted from Artemisia annua L., has been widely used in the treatment of malaria due to the characteristics of high efficiency and low toxicity, and has saved millions of lives in the past 40 years (Correa et al., 2019). In recent years, it has been reported to treat inflammation, immune regulation, 
anti-fibrosis, and other pathological processes (PaciosMichelena et al., 2021; Surowiak et al., 2021; Zhang et al., 2021b). Dihydroartemisinin (DHA) is a derivative of artemisinin and harbors nearly 10 times bioavailability as much as that of artemisinin. It ameliorated the acute kidney injury caused by immune storm and inhibited COVID-19 proliferation by targeting cell replication cycle (Uckun et al., 2021). Artemisinin was also effective in preventing the complications of heart, kidney and liver caused by lipotoxicity (Jiang et al., 2020). It inhibited inflammatory reaction and thus protected islet $\beta$-cells, improved insulin resistance, induced islet $\alpha$-cells to transform into $\beta$-cells, alleviated renal and cardiac fibrosis process, and reduced urinary protein excretion (Deng et al., 2016; Lee et al., 2012). Diabetic cardiomyopathy was often accompanied by left ventricular diastolic dysfunction, whole heart hypertrophy, arrhythmia, systolic dysfunction, and heart failure (Xiong et al., 2021). Artemisinin reversed the fibrosis process of the interstitial and peripheral blood vessels of diabetic cardiomyopathy, improved the energy metabolism composition, reduced the oxidative and inflammatory reactions, and declined mitochondrial damage (Dolivo et al., 2021). However, the mechanism of artemisinin in cardiac lipotoxicity need to be further elucidated.

MicroRNA, also known as microRNA or miRNA, is a class of non-coding single stranded RNA with only 22 nucleotide sequences. A single miRNA can recognize hundreds of different mRNA, and there is also the phenomenon that multiple miRNAs jointly regulate one mRNA (Huntington et al., 2021; Mellis and Caporali, 2018). MiRNAs are involved in the regulation of a variety of physiological and biological processes in life, including the occurrence and outcome of tumors, the regulation of immune diseases, the regulation after virus interference, and the regulation of metabolism (Mellis and Caporali, 2018). MiRNA-133b has been reported to be involved in the regulation of multiple life processes, including myocardial cells against hypoxia and infarction, but the mechanism of its action is not clear (Pan et al., 2018). Whether DHA can regulate miRNA-133b and play a protective role in myocardial cells in lipotoxicity environment deserves further discussion.

Silent information regulator 1 (Sirt1) is a nicotinamideadenine dinucleotide $\left(\mathrm{NAD}^{+}\right)$-dependent deacetylase involved in manipulating gene expression, energy metabolism, DNA damage repair and mitochondrial functions (Abraham et al., 2019; Alcendor et al., 2007; Tang, 2016). Lots of transcription factors were identified as its targets, such as p53 (Vaziri et al., 2001), FoxO family members (Hariharan et al., 2010), and PGC-1a (Dominy et al., 2010). Previous studies demonstrated that Sirt1 executed fundamental roles in influencing cell activities, such as metabolic flexibility (Wei et al., 2019), oxidative damage (Liang et al., 2020), mitochondrial dysfunction (Zhang et al., 2018), cell proliferation and apoptosis (Wu et al., 2018), and inflammation (Mirshafa et al., 2020). Piles of evidences illustrated that Sirt1 ameliorated Sodium fluoride and ischemia/perfusioninduced cell injury by inhibiting inflammatory response and apoptosis (Peng et al., 2019; Wu et al., 2018). Nonetheless, further research is in need to elucidate whether Sirtl takes part in the process of DHA in antagonizing palmitate-induced $\mathrm{H} 9 \mathrm{c} 2$ cell injury.

\section{Materials and Methods}

\section{Cell culture and drug treatment}

H9c2 cells, derived from embryonic rat heart, were purchased from the Institute of Biochemistry and Cell Biology, Chinese Academy of Sciences (Shanghai, China) and cultured in DMEM high glucose with $10 \%$ fetal bovine serum (FBS) (Life Technologies, Ballenger Creek, MD, USA) and $1 \%$ penicillin and streptomycin in $10-\mathrm{cm}$ diameter dish (Corning incorporated, Corning, NY, USA) at $37^{\circ} \mathrm{C}$ in a humid air with $5 \% \mathrm{CO}_{2}$. Cells ( ${ }^{\text {rd }}$ passage) were trypsinized at $80 \%$ confluency and subculture in the ratio of $1: 3$. Cell suspensions were used in the flowing experiments.

Cells were seeded in a 24-well plate and incubated with palmitate (PAL) (Sigma-Aldrich, St. Louis, MO, USA) at final concentration of $0.5 \mathrm{mM}$ at $50 \%$ confluence. Dihydroartemisinin (DHA) (Cat No. D831931, CAS: 71939-50-9, Formula = $\mathrm{C}_{15} \mathrm{H}_{24} \mathrm{O}_{5}, \mathrm{MW}=235.84$, purity over $98 \%$, density $=1.24 \mathrm{~g} / \mathrm{cm}^{3}$ ) was the active metabolite of artemisinin compounds and was purchased from MACKLIN company (Shanghai, China). The powder was dissolved in dimethyl sulfoxide (DMSO) and diluted with PBS or DMEM to the final concentration of $10 \mu \mathrm{M}$. The miR-133b mimic and scramble fragments were provided by Ribobio Company (Guangzhou, China). Plasmids of pcDNA3.1Sirtl and the compartment backbone vector were kindly gifted by Dr. Yinchuan Li's lab at Nantong University. Cells were ectopically transfected with plasmids or miRNAs with lipofectamine 3000 (ThermoFisher Scientific, Waltham, MA, USA) based on the manufacturer's directions.

\section{MTT assay}

MTT assay were used to investigate the cell viability. Briefly, the single cell suspensions were located in 96-well plates at about $5 \times 10^{3}$ cells/well, and $0.1 \mathrm{mg}$ MTT (Sigma-Aldrich) was added to each well. Cells then were cultured at $37^{\circ} \mathrm{C}$ in the dark for extra $4 \mathrm{~h}$. Then, $150 \mu \mathrm{L}$ DMSO was added to each well after aspiration of supernatant medium. The mixture was incubated on a rotator for $15 \mathrm{~min}$, absorbance at $490 \mathrm{~nm}$ of each well was detected by a microplate reader (Thermo Fisher Scientific, Waltham, MA; Varioskan Flash). The data were collected and calculated with the subsequent formula.

Viability rate $(\%)=100 \times$

(OD value of experimental group/OD value of control group).

Quantitative reverse transcription polymerase chain reaction ( $q R T-P C R)$

Cells were harvested and total RNA was isolated with TRIzol reagent (Vazyme, Nanjing, China) under the directions of manufacturer. RNA pellets were dissolved and quantified with a spectrophotometer (NanoDrop 2000, Thermo Fisher Scientific) and then reverse-transcribed with PrimeScript ${ }^{\mathrm{TM}}$ RT Master Mix (Takara, Shiga Prefecture, Japan, PR036A). Quantitative real-time PCR (qRT-PCR) was performed with SYBR Premix Ex Taq (Tli RNaseH Plus) (Takara, DRR420A). Measurements were carried out in triplicates and data were normalized to endogenous GAPDH expression. Primers were designed by Pick Primers software (National Center for Biotechnology Information, MD, USA) and validated. The sequences of primers used are listed in Table 1. 
TABLE 1

Primer sequence of target genes

\begin{tabular}{lll}
\hline Gene name & Forward primer & Reverse primer \\
\hline GAPDH & CCTCAAGATTGTCAGCAAT & CCATCCACAGTCTTCTGAGT \\
Casp3 & ATGTCGATGCAGCTAACCTC & TCCTTTTGCTGTGATCTTCC \\
Bax & TGCAGAGGATGATTGCTGAC & GATCAGCTCGGGCACTTTAG \\
Bcl-2 & GGGATGCCTTTGTGGAACTA & CTCACTTGTGGCCCAGGTAT \\
Sirt1 & TTATGCTCGCCTTGCTGTGG & ATGCTTCAATGCTGTTTCTTCTTTG \\
\hline & & \\
\hline Gene & Forward Primer & \\
& Sequence (5'- 3') & \\
U6 snRNA & TGGCCCCTGCGCAAGGATG & \\
miR-133b & TTTGGTCCCCTTCAACCAGCTA & \\
\hline
\end{tabular}

The expression of each gene was defined as the fold change compared with the threshold cycle $(\mathrm{Ct})$, and relative expression levels were calculated using the $2^{-\triangle \Delta \mathrm{Ct}}$ method by normalization to the housekeeping gene GAPDH or U6 snRNA. Results are presented as the means from three individual experiments.

\section{Western blot}

Total protein was prepared from the cell lysates and then the concentration was determined by the BCA protein assay kit (Thermo Fisher Scientific). Equal allocates of denatured protein were loaded into 10\% SDS-PAGE (Epizyme, Shanghai, China) and transferred to polyvinylidene difluoride (PVDF) membranes (Millipore, Billerica, MA, USA). Membranes were blocked with $5 \%$ skim milk solution and incubated with antiSirt1 (ab110304), anti-caspase 3 (ab13847), anti-Bcl-2 (ab692), anti-Bax (ab32503) or anti-GAPDH (ab181602) at $4^{\circ} \mathrm{C}$ overnight. The washed membranes were incubated with HRP conjugated secondary antibodies. Bands of targeted proteins were visualized with ECL Super Signal (Pierce, Rockford, IL, USA). Images were taken using a Tanon 5200 system (Shanghai, China) and analyzed by ImageJ software (NIH, NY, USA), using GAPDH as an internal control.

\section{Dual luciferase activity assay}

Cells $\left(5 \times 10^{3}\right.$ cells $\left./ \mathrm{mL}\right)$ were seeded on 24 -well plates and transfected with predesigned molecules (Scramble, miR133b mimic, 3'-UTR wild-type and mutant; Ribobio, China) with lipofectamine 3000 , or incubated with DHA at a final concentration of $10 \mu \mathrm{M}$. Cells were washed with PBS and then supplied with passive lysis buffer (PLB) lysate and then added Luciferase Assay Reagent (LAR, Promega, Madison, WI, USA). Dual luciferase activity assay was carried out to detect the fluorescence value in ahead of addition of stop buffer. The fluorescence data were checked again and then calculated the relative luciferase activity.

\section{Statistical analysis}

Statistical analysis was performed using SPSS Statistics 24.0 (SPSS Inc., Chicago, IL, USA). All data are presented as mean \pm standard deviation (SD), and the significant differences were analyzed by Student's $t$-test and analysis of variance (ANOVA) with Tukey's multiple comparisons test, with significance measured at ${ }^{\star} P<0.05,{ }^{* *} P<0.01$ or ${ }^{* * *} P$ $<0.001$. Pictures were processed with ImageJ software for gray density analysis and drew with GraphPad Prism 8.0.

\section{Results}

Cell viability and apoptotic gene expression after PAL treatment H9c2 cells were treated with $0.5 \mathrm{mM}$ PAL for $12,24,48$, and $72 \mathrm{~h}$. Results showed that PAL administration lowered the cell viability, as assessed by MTT assay, and significant difference was observed at $24 \mathrm{~h}(P<0.01$, Fig. $1 \mathrm{~A}), 48 \mathrm{~h}(P<0.001$, Fig. 1A), and $72 \mathrm{~h}(P<0.001$, Fig. 1A). Transcriptional variation of Casp3 elevated continuously after PAL incubation at $12 \mathrm{~h}(P<0.05$, Fig. $1 \mathrm{~B}), 24 \mathrm{~h}(P<0.01$, Fig. 1B), $48 \mathrm{~h}(P<0.01$, Fig. $1 \mathrm{~B})$, and $72 \mathrm{~h}(P<0.001$, Fig. 1B). While expression of $\mathrm{Bcl}-2$ exhibited no difference after PAL treatment $(P>0.05$, Fig. 1C). Changes of Bax escalated persistently after PAL stimulation at $12 \mathrm{~h}(P<0.01$, Fig. 1D), $24 \mathrm{~h}(P<0.001$, Fig. 1D), $48 \mathrm{~h}(P<0.001$, Fig. 1D), and $72 \mathrm{~h}(P<0.001$, Fig. 1D). Protein expression was investigated, and results showed that Caspase3 $(12 \mathrm{~h}, P<$ $0.05 ; 24$ h, $P<0.001 ; 48$ h, $P<0.001 ; 72$ h, $P<0.001$. Figs. $1 \mathrm{E}$ and $1 \mathrm{~F})$ and $\operatorname{Bax}(12 \mathrm{~h}, P>0.05 ; 24 \mathrm{~h}, P<0.001 ; 48 \mathrm{~h}, P$ $<0.001 ; 72 \mathrm{~h}, P<0.001$. Figs. $1 \mathrm{E}$ and $1 \mathrm{~F})$ exhibited the same pattern as its gene expression, while Bcl-2 reached its peak at $12 \mathrm{~h}$ and then declined as time went by $(12 \mathrm{~h}, P<0.01 ; 24 \mathrm{~h}$, $P<0.01 ; 48$ h, $P<0.01 ; 72$ h, $P>0.05$. Figs. $1 \mathrm{E}$ and $1 \mathrm{~F})$. These results indicated that PAL incubation resulted in cell injury in $\mathrm{H} 9 \mathrm{c} 2$ and promoted the apoptotic genes expression.

\section{DHA attenuated PAL-induced H9c2 injury}

The administration of DHA $(10 \mu \mathrm{M})$ hindered the PALaroused cell injury in $\mathrm{H} 9 \mathrm{c} 2$ as detected by the cell viability variations. DHA increased cell viability in PAL-stimulated cells at $48 \mathrm{~h}(P<0.05$, Fig. $2 \mathrm{~A})$ and $72 \mathrm{~h}(P<0.001$, Fig. 2A). Moreover, PAL enhanced expression level of Casp3 (PAL vs. Control, $P<0.001$, Fig. $2 \mathrm{~B}$ ) and lowered by the administration of DHA (DHA vs. PAL, $P<0.01$, Fig. $2 \mathrm{~B}$ ). Both drugs showed no effects on the transcription of $\mathrm{Bcl}-2$ 


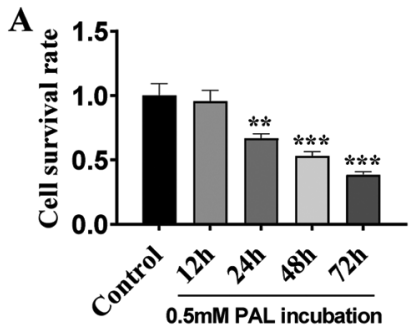

D

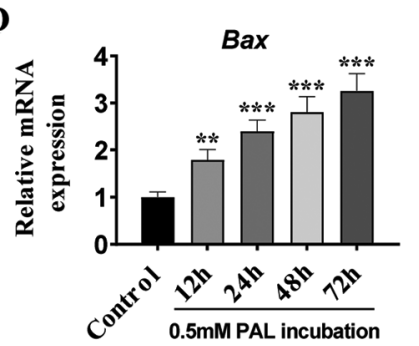

B

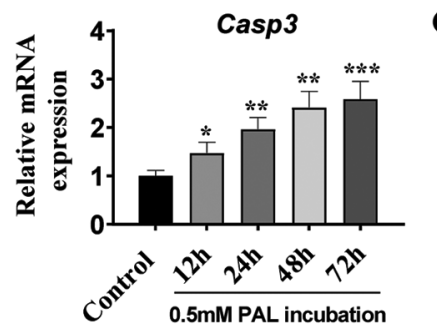

E

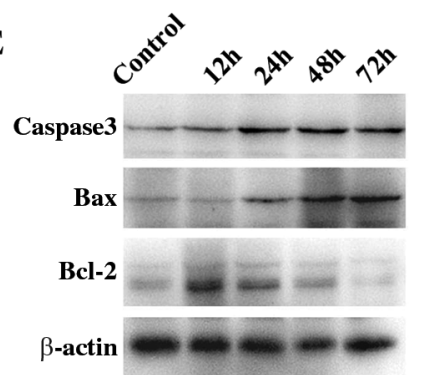

C

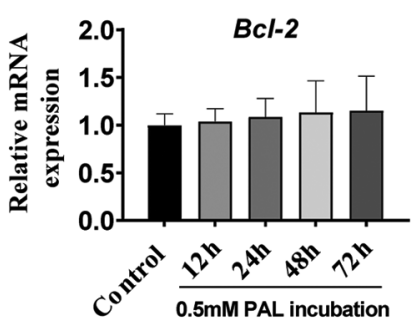

F

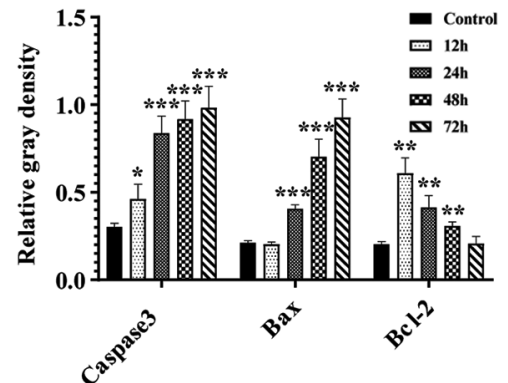

FIGURE 1. Palmitate incubation resulted in H9c2 cell injury. (A) Cell survival rate. (B-D) Transcriptional expression of Casp3, Bcl-2, and Bax detected by qRT-PCR. (E) Protein expression of Caspase3, Bax, and Bcl-2 examined by western blot. (F) Statistical analysis of the western blot results based on relative gray density. The final concentration of PAL was $0.5 \mathrm{mM}$. N = 7 for cell survival investigation, $\mathrm{N}=8$ for $\mathrm{mRNA}$ detection, and $\mathrm{N}=3$ for western blot analysis. "PAL" was short for "palmitate" in the figure. ${ }^{\star} P<0.05,{ }^{* *} P<0.01,{ }^{* * *} P<0.001$.

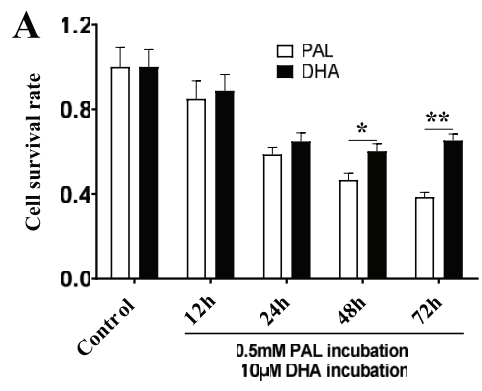

D

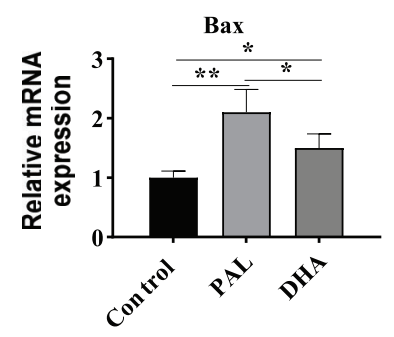

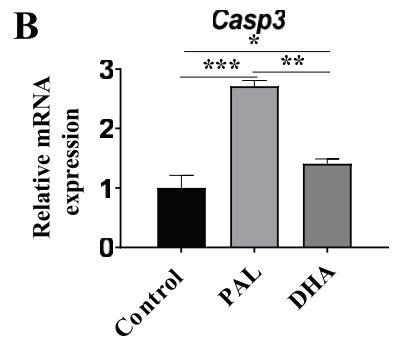

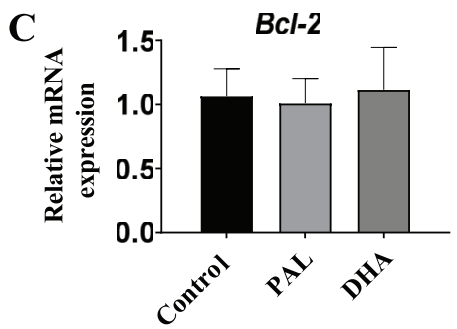

$\mathbf{E}$

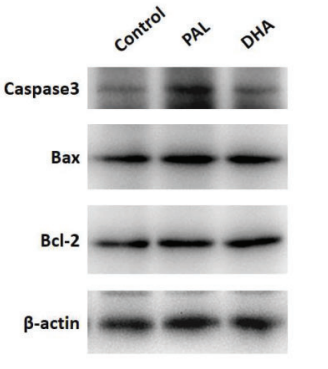

$\mathbf{F}$

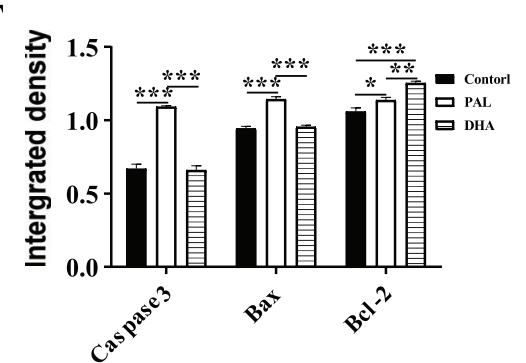

FIGURE 2. DHA alleviated palmitate induced cardiomyocytes injury. (A) Cell survival rate after treatment of DHA by MTT assay. (B-D) Casp3, Bax, and Bcl-2 mRNA expression. (E) Western blot analysis of Caspase 3, Bax, and Bcl-2. (F) Gray density analysis for results of panel E. The final concentration of PAL was $0.5 \mathrm{mM}$, and DHA was $10 \mu \mathrm{M} . \mathrm{N}=7$ for cell survival investigation, $\mathrm{N}=8$ for mRNA detection, and $\mathrm{N}=$ 3 for western blot analysis. "DHA" was short for "Dihydroartemisinin" in the figure and cells in this group would be pre-incubated with PAL. ${ }^{\star} P<0.05,{ }^{* *} P<0.01,{ }^{* *} P<0.001$.

( $P>0.05$, Fig. 2C). Variations of Bax was boosted by PAL (PAL vs. Control, $P<0.01$, Fig. 2D) and lowered by DHA (DHA vs. PAL, $P<0.05$, Fig. 2D), while the value was still higher than that in control group (DHA vs. Control, $P<$ 0.05 , Fig. 2D). Expression of proteins were discovered, and data exhibited that PAL enhanced the content of Caspase 3 (PAL vs. Control, $P<0.001$, Figs. $2 \mathrm{E}$ and $2 \mathrm{~F}$ ) while abolished by administration of DHA (DHA vs. PAL, $P<$ 0.001 , Figs. $2 \mathrm{E}$ and $2 \mathrm{~F}$ ). Similar pattern was observed in expression of Bax protein (PAL vs. Control and DHA vs. PAL, $P<0.001$, Figs. $2 \mathrm{E}$ and $2 \mathrm{~F})$. Bcl-2 expression was enhanced by the addition of DHA (DHA vs. Control, $P<$
0.001; DHA vs. PAL, $P<0.01$, Figs. $2 \mathrm{E}$ and $2 \mathrm{~F}$ ). Data above demonstrated that DHA reversed PAL-induced cell injury and protected cells from the attack of apoptotic gene cascade.

DHA decreased the expression of $\mathrm{miR}-133 \mathrm{~b}$ in cardiomyocytes treated with $P A L$

Real time quantitative PCR was used to analyze the expression of miR-133b in cardiomyocytes treated with PAL. The results showed that the expression of miR-133b in cardiomyocytes increased significantly after treatment with PAL (PAL vs. Control, $P<0.001$, Fig. 3A), while decreased by DHA incubation (PAL vs. DHA, $P<0.01$, Fig. $3 \mathrm{~A}$ ). During the 
time course investigation, PAL incubation enhanced the expression of miR-133b significantly compared to control group (12 h, $P<0.01 ; 24 \mathrm{~h}, P<0.001 ; 48 \mathrm{~h}, P<0.001 ; 72 \mathrm{~h}$, $P<0.01$, Fig. 3B). To investigate the efficiency of abnormal expression of miR-133b, the mimic and antagomir fragments were introduced into $\mathrm{H} 9 \mathrm{c} 2$ cells. As shown in Fig. 3C, mimic sequence drastically promoted the ectopic expression of miR-133b (miR-133b mimic vs. Scramble, $P<$ 0.001 ) and the basic expression of miR-133b was abolished by the siRNA (siRNA miR-133b vs. Scramble, $P<0.01$ ). Furthermore, PAL downregulated the expression of miR133b (Blank vs. Control, $P<0.01$, Fig. 3C), while DHA upregulated its expression (DHA vs. Blank, $P<0.05$, Fig. 3C). In addition, mimic fragments transfection evidently inhibited the cell viability (miR-133b mimic vs. Scramble, $P<0.05$, Fig. 3D), while miR-133b inhibitor enhanced the survival rate of cells (siRNA miR-133b vs. Scramble, $P<0.05$, Fig. 3D). In summary, the abovementioned data hinted that DHA protected H9c2 cells from PAL-induced injury by downregulation of miR-133b.

\section{DHA enhanced Sirt1 expression}

Software on miRNAs, such as TargetScan (http://www. targetscan.org/vert_72/), and miRDB (http://mirdb.org/), were adopted into the scooping of targets of miR-133b. Sirt1 (Silent information regulator I) was picked out for further research and its transcriptional changes were investigated by qRT-PCR. Findings showed that palmitate suppressed the expression of Sirt1 (PAL vs. Control, $P<0.01$, Fig. 4A), while enhanced by the administration of DHA (DHA vs. Control, $P<0.001$, Fig. 4A). Protein expression determined
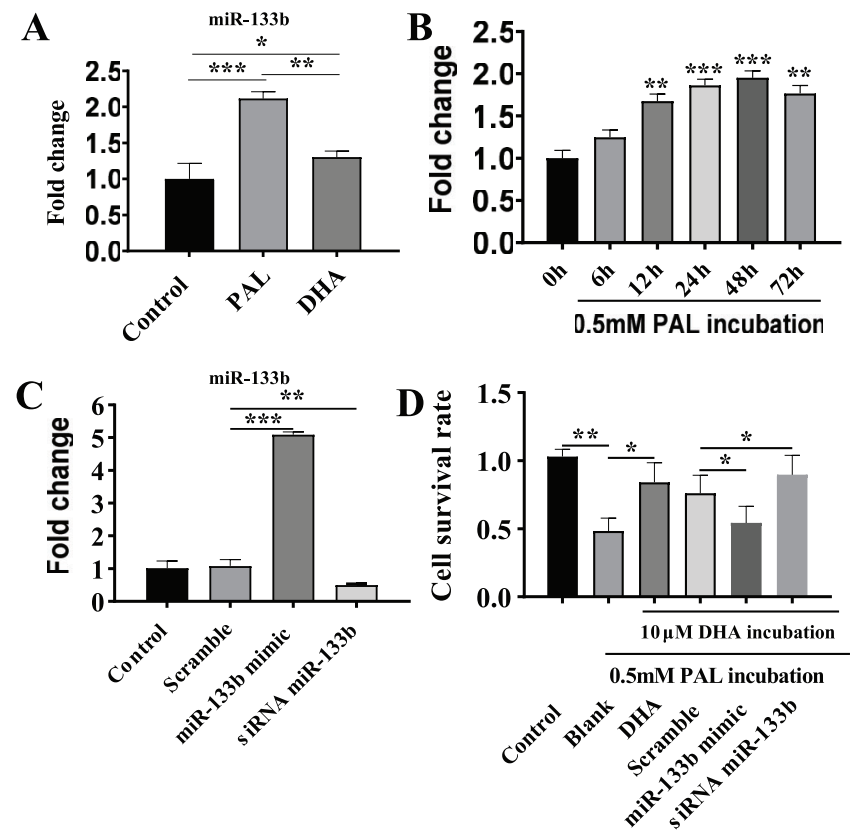

FIGURE 3. DHA decreased expression of miR-133b in cardiomyocytes. (A) Expression of miR-133b after DHA incubation. (B) Expression pattern of miR-133b at different time after $0.5 \mathrm{mM}$ PAL incubation. (C) Ectopic expression of miR-133b and knockdown efficient of miR-133b antagomir checked by qRTPCR. (D) Cell survival rate examination. The final concentration of PAL was $0.5 \mathrm{mM}$, and DHA was $10 \mu \mathrm{M}$. $\mathrm{N}=7$ for cell survival investigation and $\mathrm{N}=8$ for mRNA detection. by western blot illustrated similar pattern as PAL significantly lowered Sirtl expression (PAL vs. Control, $P<$ 0.01 , Figs. $4 \mathrm{~B}$ and $4 \mathrm{C}$ ) and DHA escaladed its content (DHA vs. Control, $P<0.001$, Figs. $4 \mathrm{~B}$ and $4 \mathrm{C}$ ). These data suggested that Sirt1 might also be involved in the functions of DHA in cell behavior.

\section{Sirt1 was a target of $\mathrm{miR}-133 \mathrm{~b}$}

Extra experiments were designed to reveal the potential relationship between miR-133b and Sirt1. Based on the analysis of the miRNA databases, miR-133b was predicted to directly bind to the 3'-UTR of Sirt1 mRNA (Fig. 5A). To confirm the physical interaction of the prediction, luciferase assay was taken out. As shown in Fig. 5B, the relative luciferase activity was reduced in the wild-type Sirt1 3'-UTR plasmid and miR-133b mimic co-transfected group $(P<$ 0.01 , Fig. 5B). However, no significant difference was investigated in Sirt1 mutant groups (Fig. 5B). Moreover, the expression of Sirt1 was downregulated by miR-133b overexpression compared to scramble group $(P<0.001$, Figs. 5C and 5D). Collectively, these results demonstrated that Sirt1 was a target of miR-133b.

DHA ameliorated PAL-induced cell injury via increasing of Sirt1

Ectopically overexpressed Sirt1 by transfection boosted its content in H9c2 cells (pcDNA3.1-Sirt1 vs. pcDNA3.1, $P<$ 0.001 , Fig. $6 \mathrm{~A}$ ), and miR-133b addition reduced the abnormal expression of Sirt1 (pcDNA3.1-Sirt1+miR-133b mimic vs. pcDNA3.1-Sirt1, $P<0.01$, Fig. 6A). Moreover, plasmids transfection enhanced the protein expression of Sirt1 (pcDNA3.1-Sirt1 vs. pcDNA3.1, $P<0.001$, Figs. 6B and 6C) and decreased by the addition of miR-133b mimic (pcDNA3.1-Sirt1+miR-133b mimic vs. pcDNA3.1-Sirt1, $P<$ 0.01 , Figs. $6 \mathrm{~B}$ and $6 \mathrm{C}$ ). In addition, adoption of abnormal Sirt1 overexpression improved the cell viability (pcDNA3.1Sirt1 vs. pcDNA3.1, $P<0.05$, Fig. 6D), while decreased by the addition of miR-133b (pcDNA3.1-Sirt1+miR-133b mimic vs. pcDNA3.1-Sirt1, $P<0.05$, Fig. 6D). Taken together, data collected above indicated that DHA ameliorated palmitateinduced cell injury by upregulation of Sirt1.

DHA protected PAL-induced cell injury might by influencing miR-133b/Sirt1 axis

Adoption of DHA reversed the palmitate-induced cell viability reduction (DHA vs. Blank, $P<0.01$, Fig. 7A), extra expressed miR-133b partially abolished the effect (miR-133b mimic $v s$. Scramble, $P<0.05$, Fig. 7A). Ectopically overexpressed Sirt1 synergically promoted the cell viability (pcDNA3.1-Sirt1 vs. pcDNA3.1, $P<0.05$, Fig. 7A), while the administration of miR-133b declined the cell survival rate (pcDNA3.1-Sirt1 + miR-133b mimic vs. pcDNA3.1-Sirt1, $P<0.05$, Fig. 7A). Additionally, luciferase report assay was taken to reveal the regulatory relationship between DHA and miR-133b/Sirt1 axis. Results exhibited that miR-133b bound to wild type Sirt1 3'-UTR and declined the transcriptional ability $(P<$ 0.001 , Fig. $7 \mathrm{~B}$ ), and DHA administration partially enhanced the transcription by antagonizing the inhibition of miR-133b $(P<0.01$, Fig. 7B). On the contrary, both miR-133b and DHA showed no influence on the transcription ability in 

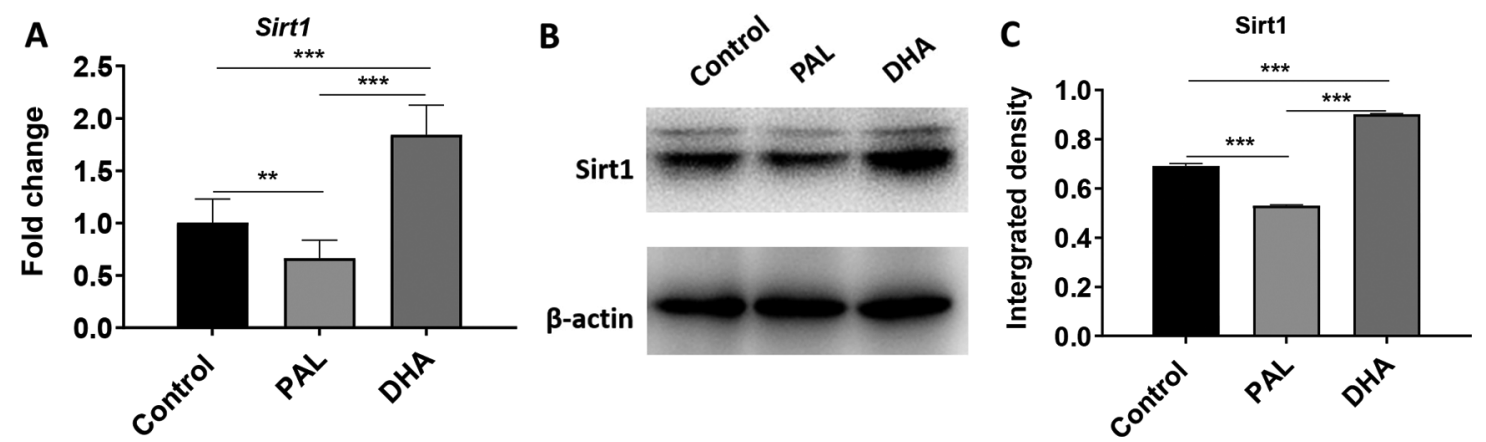

FIGURE 4. DHA upregulated expression of silent information regulator I (Sirt1). The expression of Sirt1 was checked by qRT-PCR $(A, N=8)$ and Western blot $(\mathrm{B}, \mathrm{N}=3)$. (C) Gray density analysis for results of panel $\mathrm{B} .{ }^{\star} P<0.05,{ }^{\star \star} P<0.01,{ }^{\star *} P<0.001$. The final concentration of $\mathrm{PAL}$ was $0.5 \mathrm{mM}$, and DHA was $10 \mu \mathrm{M}$.

A
WT Sirt1 3'UTR 5'
...GGAACAGCUAAUCUAGACCAAAG...
$\operatorname{miR}-133 \mathrm{~b} 3^{\prime}$
| | | | |
AUCGACCAACUUCCCCUGGUUU
| | | | |
MUT Sirt1 3'UTR 5'
GGAACAGCUAAUCUACUGGUUUG
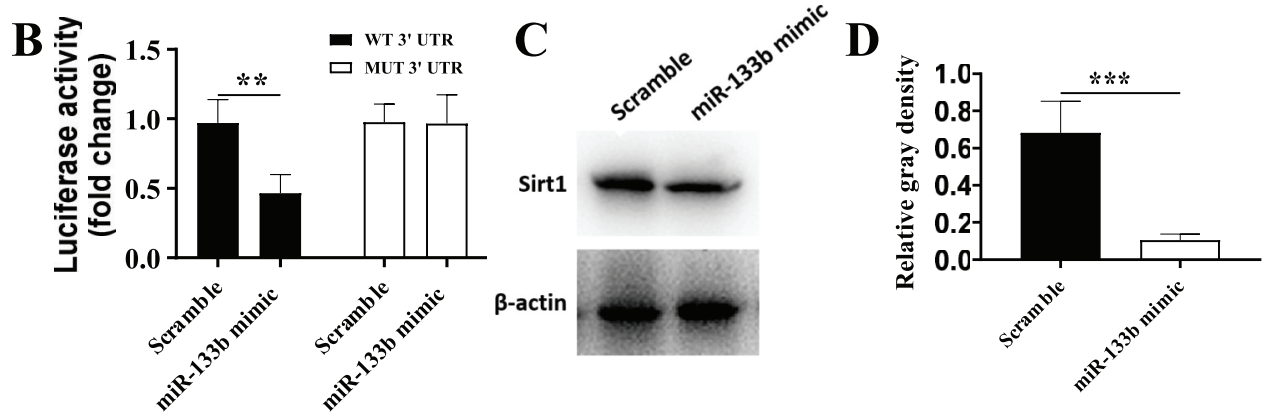

FIGURE 5. SIRT1 was a target of miR-133b. (A) Schematic diagram of predicted interacting loci of miR-133b with Sirt1 3'-UTR. (B) Luciferase assay to testify the relationship between them. (C) Expression of Sirtl under the regulation of miR-133b. (D) Gray density analysis for results of panel C. $\mathrm{N}=3 .{ }^{* *} P<0.01,{ }^{* *} P<0.001$.

A

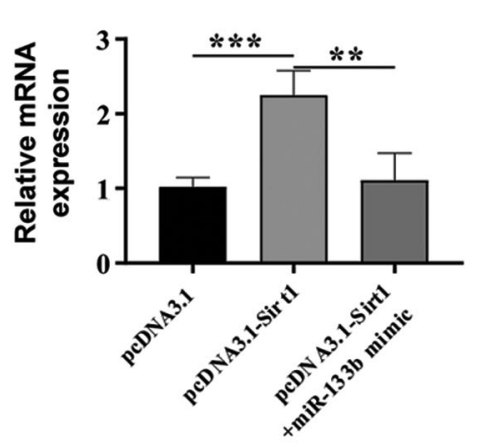

C

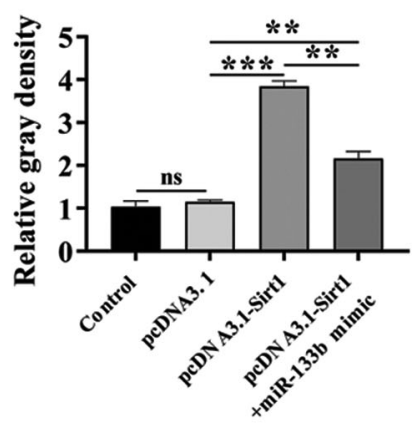

B

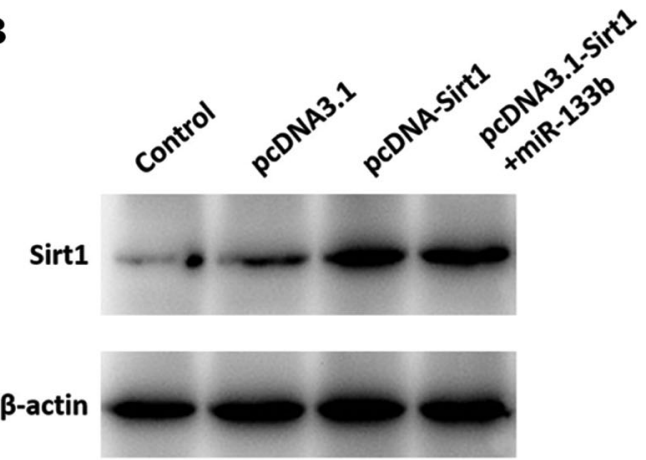

D

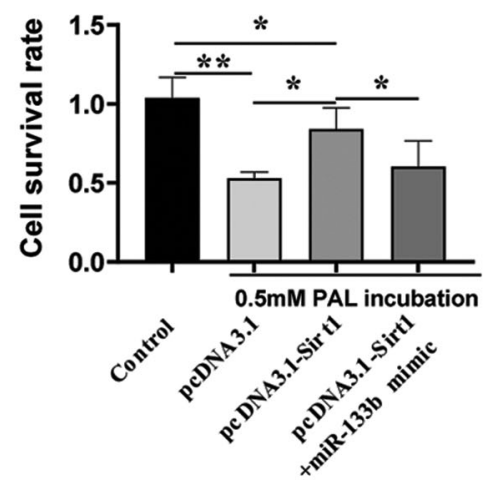

FIGURE 6. miR-133b affected cardiomyocytes survive via regulating Sirt1 expression in confronting with PAL. The expression of Sirt1 was checked by qRT-PCR (A, N = 5) and Western blot (B, N = 3). (C) Grayscale analysis for results of panel B. (D) Cell survival rate detected by MTT assay, $\mathrm{N}=7 .{ }^{*} P<0.05,{ }^{* *} P<0.01,{ }^{* *} P<0.001$. The final concentration of PAL was $0.5 \mathrm{mM}$. 


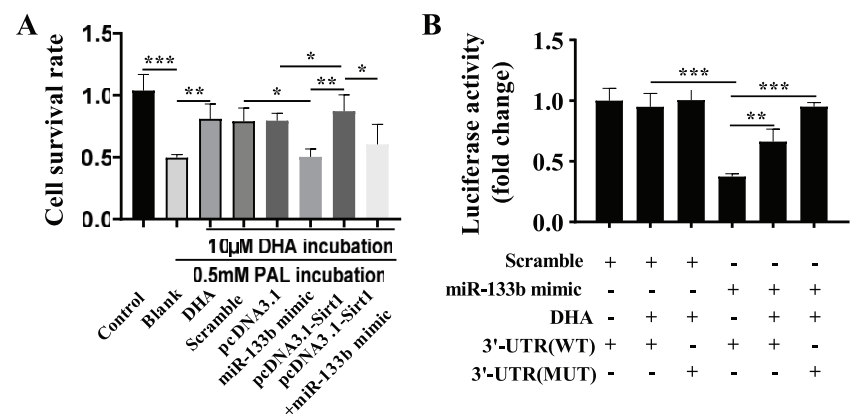

FIGURE 7. DHA protected PAL-induced cell injury by regulating miR-133b/Sirt1 axis. (A) Cell survival rate detection after Sirt1 and miR-133b overexpression under treatment of DHA by MTT assay. (B) Luciferase assay for the DHA regulation on Sirt1 transcription by targeting miR-133b. $\mathrm{N}=7$ for cell survival investigation and $\mathrm{N}$ $=3$ for Luciferase detection. ${ }^{\star} P<0.05,{ }^{*} P<0.01$, ${ }^{\star *} P<0.001$. The final concentration of PAL was $0.5 \mathrm{mM}$, and DHA was $10 \mu \mathrm{M}$.

dealing with the mutant Sirt1 3'-UTR. In summary, DHA might harbor certain inhibitory ability on miR-133b and thus upregulate the biological activity of Sirt1.

\section{Discussion}

The current study was set up to determine the roles of DHA on palmitate-treated cell viability and injury. It was exciting to pay attention that DHA ameliorated palmitate-induced cell injury as supported by enhancing cell survival while lowering apoptosis. Extra experiments evidenced that inhibition of cell injury by DHA was relied on decreasing expression of miR-133b. Furthermore, Sirt1 was mined as a target of miR-133b in this cell model and could be directly bound by miR-133b and thus declined the transcription ability. Finally, DHA protected H9c2 cells from palmitateinduced cell injury might by regulating miR-133b/Sirt1 axis.

One of the aims of this study was to set up an effective in vitro cardiomyocyte cell injury model. Obviously, after palmitate treatment, cell viability was downregulated and apoptotic genes expression was upregulated, which indicated that palmitate induced cell injury in $\mathrm{H} 9 \mathrm{c} 2$. Our study demonstrated that $0.5 \mu \mathrm{M}$ palmitate incubation suppressed cell viability significantly at $24 \mathrm{~h}$ and continuously to $72 \mathrm{~h}$, which hinted that the accumulation of fatty acid led to sustained lipotoxicity and thus resulted in cell apoptosis (Ren et al., 2021). The pro-apoptotic genes expression, including Caspase 3 and Bax, was enhanced under the stimulation of palmitate that was consistent with the results of cell viability ( $\mathrm{Xu}$ et al., 2021). The transcriptional expression of $\mathrm{Bcl}-2$ showed no variations after palmitate stimulation, while its protein expression was enhanced by palmitate administration, that contradiction may lie in the different rate of transcription, translation, and degradation of Bcl-2 (Cai et al., 2021; Wang et al., 2021a). These findings certainly noted that palmitate could induce cell injury in $\mathrm{H} 9 \mathrm{c} 2$.

Another aim of the study was to dig out the function of DHA on palmitate-induced H9c2 cell injury. Owning to its high efficiency and low toxicity, the biological activity of DHA was 10 times as much as that of artemisinin. Its antimalaria effect was 4-8 times of that of artemisinin. Results in this study demonstrated that DHA alleviated palmitateinduced cell injury with evidence in raising cell viability and lowering apoptotic genes expression. Previous study illustrated that DHA functioned in preventing palmitate-induced apoptosis in $\beta$-cells (Wang et al., 2021b). Artesunate, a kind of bioactive substance of Artemisinin, alleviated myocardial ischemia/reperfusion-induced myocardial necrosis in rats and hypoxia/reoxygenation-induced apoptosis in $\mathrm{H} 9 \mathrm{c} 2$ cells via regulating the FAK/PI3K/Akt pathway (Fan et al., 2020). This result evidenced that DHA, a derivative of artemisinin, harbored anti-injury functions in $\mathrm{H} 9 \mathrm{c} 2$ cells.

Artemisinin is a kind of active ingredient purified from Artemisia annua, a traditional Chinese herbal medicine. The functions of artemisinin mainly focus on the regulation of allergy, anti-allergy, immune regulation and fever suppression and detoxification (Çapci et al., 2021). Recent studies have shown that artemisinin has a certain effect on systemic lupus erythematosus (Chen et al., 2021; Lin et al., 2021). Artemisinin exhibited anti-inflammatory activity and lowered the systemic levels of inflammatory cytokines that resulted in cytokine storm and inflammatory organ injury in high-risk COVID-19 patients (Uckun et al., 2021). However, the role of DHA in regulating cell injury and the certain mechanism was less reported. Plenty of miRNAs were introduced to be involved in the cardiomyocytes' injury and apoptosis. MiR133b was a tumor suppressor (Cheng et al., 2021; Hu et al., 2021). In addition, it was revealed that miR-133b played important roles in various diseases. MiR-133b may play a crucial role in suppression genes associated with humans and mice cleft lip (Yoshioka et al., 2021), and act as biomarker in cardiovascular disease (Chang et al., 2021), muscle atrophy (Malacarne et al., 2021), muscle damage (Chalchat et al., 2021). Moreover, lots of reports demonstrated that miR-133b harbored the ability in cardiomyocyte protection in dealing with hypoxia injury (Pan et al., 2018; Zhou et al., 2019) and morphine preconditioning (He et al., 2016). In this study, DHA decreased miR-133b expression and extra experiments were executed to validate the relationship that DHA might exert the protection role via downregulation of miR-133b. MiR-133b overexpression declined cell viability and DHA administration abolished the effects of the molecule. Thus, our findings enriched the roles of miR-133b in cardiomyocyte protection in confronting with palmitate.

In addition, the relationship between miR-133b and Sirt1 has been revealed in former literature on bladder cancer (Chen et al., 2020), diabetic nephropathy (Sun et al., 2018), osteosarcoma (Shi et al., 2017) and glioma (Li et al., 2016) and hepatocellular carcinoma (Tian et al., 2016). In this study, experiments illustrated that DHA increased expression of Sirt1 which hinted that Sirt1 might play certain role in cardiomyocyte protection in palmitate challenge. Similarly, the regulatory relationship between miR-133b and Sirtl was confirmed and consistent with the previous studies listed above. Extra experiments illustrated that knockdown expression of Sirt1 blunted the effects of DHA in antagonizing palmitate-induced cardiomyocyte injury. Our findings suggested that DHA functions through miR-133b/Sirt1 axis.

In summary, Sirt1 was downregulated in $\mathrm{H} 9 \mathrm{c} 2$ in palmitate treatment, and this effect could be reversed by DHA incubation. 
Similarly, miR-133b was increased in palmitate-induced H9c2 cells, which directly suppressed the expression of Sirt1. DHA exerted cardiomyocyte protection in dealing with lipotoxicity by regulating the miR-133b/Sirt1 axis in palmitate-treated H9c2 cells. Our findings enriched extra evidence of the mechanisms of DHA and its function in the protection of cardiomyocyte injury. Therefore, further studies were in demanding to clarify the mechanism and to promote the rationale for its clinical use.

Acknowledgement: We appreciate Dr. Yinchuan Li for kindly gifting us the plasmids in this study and other participants who support us in the research.

Availability of Data and Materials: The datasets enrolled and analyzed in this study are available from the corresponding author on reasonable request.

Authors' Contribution: The current work was performed by all authors in collaboration. QW and XZ designed the project; LQ, BL, BC, XX and SW devoted to the experiments; LQ, CL and XX participated in analyzing the data; LQ, XX and QW wrote the manuscript.

Funding Statement: This work was supported by Nantong Science and Technology Project (MS12018020, MS12018041); Jiangsu Government Scholarship for Overseas Studies (JS2017-200), the Doctoral Scientific Research Foundation of Nantong University (135420505015) and the Natural Science Foundation of the Jiangsu Higher Education Institutions of China (11KJB180010).

Conflicts of Interest: The authors declare that they have no conflicts of interest to report regarding the present study.

\section{References}

Abraham A, Qiu S, Chacko BK, Li H, Paterson A et al. (2019). SIRT1 regulates metabolism and leukemogenic potential in CML stem cells. Journal of Clinical Investigation 129: 2685-2701.

Alcendor RR, Gao S, Zhai P, Zablocki D, Holle E et al. (2007). Sirt1 regulates aging and resistance to oxidative stress in the heart. Circulation Research 100: 1512-1521.

Cai X, He L, Zhou G, Li S, Liao X (2021). Mogroside IIe ameliorates cardiomyopathy by suppressing cardiomyocyte apoptosis in at type 2 diabetic model. Frontiers in Pharmacology 12: 650193.

Çapcı A, Herrmann L, Sampath Kumar H, Fröhlich T, Tsogoeva S (2021). Artemisinin-derived dimers from a chemical perspective. Medicinal Research Reviews 41: 2927-2970. DOI 10.1002/med.21814.

Correa SAP, de Oliveira RN, Mendes TMF, Dos Santos KR, Boaventura S Jr. et al. (2019). In vitro and in vivo evaluation of six artemisinin derivatives against Schistosoma mansoni. Parasitology Research 118: 505-516.

Chalchat E, Charlot K, Garcia-Vicencio S, Hertert P, Baugé S et al. (2021). Circulating microRNAs after a 24-h ultramarathon run in relation to muscle damage markers in elite athletes. Scandinavian Journal of Medicine \& Science in Sports 31: 1782-1795. DOI 10.1111/sms.14000.

Chang Y, Tuz-Zahra F, Godbole S, Avitia Y, Bellettiere J et al. (2021). Endothelial-derived cardiovascular disease-related microRNAs elevated with prolonged sitting pattern among postmenopausal women. Scientific Reports 11: 11766. DOI 10.1038/s41598-02190154-1.
Chen J, Li Y, Li Z, Cao L (2020). LncRNA MST1P2/miR-133b axis affects the chemoresistance of bladder cancer to cisplatinbased therapy via Sirt1/p53 signaling. Journal of Biochemical and Molecular Toxicology 34: e22452. DOI 10.1002/jbt.22452.

Chen Y, Tao T, Wang W, Yang B, Cha X (2021). Dihydroartemisinin attenuated the symptoms of mice model of systemic lupus erythematosus by restoring the Treg/Th17 balance. Clinical and Experimental Pharmacology and Physiology 48: 626633. DOI 10.1111/1440-1681.13461.

Cheng J, Ma H, Yan M, Xing W (2021). THAP9-AS1/miR-133b/ SOX4 positive feedback loop facilitates the progression of esophageal squamous cell carcinoma. Cell Death \& Disease 12: 401. DOI 10.1038/s41419-021-03690-z.

Deng Y, Matsui Y, Pan W, Li Q, Lai ZC (2016). Yap1 plays a protective role in suppressing free fatty acid-induced apoptosis and promoting beta-cell survival. Protein \& Cell 7: 362-372. DOI 10.1007/s13238-016-0258-5.

Dominy JE Jr., Lee Y, Gerhart-Hines Z, Puigserver P (2010). Nutrient-dependent regulation of PGC-1alpha's acetylation state and metabolic function through the enzymatic activities of Sirt1/GCN5. Biochimica et Biophysica Acta 1804: 1676-1683. DOI 10.1016/j.bbapap.2009.11.023.

Dolivo D, Weathers P, Dominko T (2021). Artemisinin and artemisinin derivatives as anti-fibrotic therapeutics. Acta Pharmaceutica Sinica B 11: 322-339. DOI 10.1016/j.apsb.2020.09.001.

Fan S, Zhang D, Liu F, Yang Y, Xu H (2020). Artesunate alleviates myocardial ischemia/reperfusion-induced myocardial necrosis in rats and hypoxia/reoxygenation-induced apoptosis in H9C2 cells via regulating the FAK/PI3K/Akt pathway. Annals of Translational Medicine 8: 1291. DOI 10.21037/atm-20-5182.

Hariharan N, Maejima Y, Nakae J, Paik J, Depinho RA, Sadoshima J (2010). Deacetylation of FoxO by Sirt1 plays an essential role in mediating starvation-induced autophagy in cardiac myocytes. Circulation Research 107: 1470-1482. DOI 10.1161/CIRCRESAHA.110.227371.

He S, Zhu H, Han Z, Wu H, Jin S et al. (2016). MicroRNA-133b-5p is involved in cardioprotection of morphine preconditioning in rat cardiomyocytes by targeting Fas. Canadian Journal of Cardiology 32: 996-1007. DOI 10.1016/j.cjca.2015.10.019.

Heallen T, Kadow Z, Kim J, Wang J, Martin J (2019). Stimulating cardiogenesis as a treatment for heart failure. Circulation Research 124: 1647-1657. DOI 10.1161/CIRCRESAHA.118.313573.

Hu C, Wu J, Wang L, Liu X, Da B et al. (2021). miR-133b inhibits cell proliferation, migration, and invasion of lung adenocarcinoma by targeting CDCA8. Pathology Research and Practice 223: 153459. DOI 10.1016/j.prp.2021.153459.

Huntington J, Pachauri M, Ali H, Giacca M (2021). RNA interference therapeutics for cardiac regeneration. Current Opinion in Genetics \& Development 70: 48-53. DOI 10.1016/j.gde.2021.05.007.

Jiang Y, Du H, Liu X, Fu X, Li X, Cao Q (2020). Artemisinin alleviates atherosclerotic lesion by reducing macrophage inflammation via regulation of $\mathrm{AMPK} / \mathrm{NF}-\mathrm{KB} / \mathrm{NLRP} 3$ inflammasomes pathway. Journal of Drug Targeting 28: 70-79. DOI 10.1080/1061186X.2019.1616296.

Kakimoto P, Serna J, De Miranda Ramos V, Zorzano A, Kowaltowski A (2021). Increased glycolysis is an early consequence of palmitate lipotoxicity mediated by redox signaling. Redox Biology 45: 102026. DOI 10.1016/j.redox.2021.102026.

Kanamori H, Naruse G, Yoshida A, Minatoguchi S, Watanabe T et al. (2021). Morphological characteristics in diabetic cardiomyopathy associated with autophagy. Journal of Cardiology 77: 30-40. DOI 10.1016/j.jjcc.2020.05.009. 
Lee IS, Ryu DK, Lim J, Cho S, Kang BY, Choi HJ (2012). Artesunate activates Nrf2 pathway-driven anti-inflammatory potential through ERK signaling in microglial BV2 cells. Neuroscience Letters 509: 17-21. DOI 10.1016/j.neulet.2011.12.034.

Li C, Liu Z, Yang K, Chen X, Zeng Y et al. (2016). MiR-133b inhibits glioma cell proliferation and invasion by targeting Sirt1. Oncotarget 7: 36247-36254. DOI 10.18632/oncotarget.9198.

Liang D, Zhuo Y, Guo Z, He L, Wang X et al. (2020). SIRT1/PGC-1 pathway activation triggers autophagy/mitophagy and attenuates oxidative damage in intestinal epithelial cells. Biochimie 170: 10-20. DOI 10.1016/j.biochi.2019.12.001.

Lin Z, Liu Y, Chen L, Cao S, Huang Y et al. (2021). Artemisinin analogue SM934 protects against lupus-associated antiphospholipid syndrome via activation of Nrf2 and its targets. Science China Life Sciences 64: 1702-1719. DOI 10.1007/s11427-020-1840-1.

Liu Y, Zeng L, Yang Y, Chen C, Wang D, Wang H (2020). Acyl-CoA thioesterase 1 prevents cardiomyocytes from Doxorubicininduced ferroptosis via shaping the lipid composition. Cell Death \& Disease 11: 756. DOI 10.1038/s41419-020-02948-2.

Malacarne C, Galbiati M, Giagnorio E, Cavalcante P, Salerno F et al. (2021). Dysregulation of muscle-specific microRNAs as common pathogenic feature associated with muscle atrophy in ALS, SMA and SBMA: Evidence from animal models and human patients. International Journal of Molecular Sciences 22: 5673. DOI 10.3390/ijms22115673.

Mellis D, Caporali A (2018). MicroRNA-based therapeutics in cardiovascular disease: Screening and delivery to the target. Biochemical Society Transactions 46: 11-21. DOI 10.1042/ BST20170037.

Mirshafa A, Mohammadi H, Shokrzadeh M, Mohammadi E, Talebpour Amiri F, Shaki F (2020). Tropisetron protects against brain aging via attenuating oxidative stress, apoptosis and inflammation: The role of SIRT1 signaling. Life Sciences 248: 117452. DOI 10.1016/j.lfs.2020.117452.

Noronha-Dutra A, Steen E (1982). Lipid peroxidation as a mechanism of injury in cardiac myocytes. Laboratory Investigation 47: 346-353.

Pacios-Michelena A, Kasaragod VB, Schindelin H (2021). Artemisinins and their impact on inhibitory neurotransmission. Current Opinion in Pharmacology 59: 19-25. DOI 10.1016/j.coph.2021.04.008.

Pan Y, Han Z, He S, Yang W, Cheng J et al. (2018). contributes to hypoxic preconditioning-mediated cardioprotection by inhibiting the activation of caspase- 8 and caspase-3 in cardiomyocytes. Molecular Medicine Reports 17: 70977104. DOI $10.3892 / \mathrm{mmr} .2018 .8784$.

Peng W, Xu S, Zhang J, Zhang Y (2019). Vitamin C attenuates sodium fluoride-Induced mitochondrial oxidative stress and apoptosis via Sirt1-SOD2 pathway in F9 cells. Biological Trace Element Research 191: 189-198. DOI 10.1007/ s12011-018-1599-0.

Ren J, Wu NN, Wang S, Sowers JR, Zhang Y (2021). Obesity cardiomyopathy: Evidence, mechanisms and therapeutic implications. Physiological Reviews 101: 1745-1807. DOI 10.1152/physrev.00030.2020.

Sun Z, Ma Y, Chen F, Wang S, Chen B, Shi J (2018). miR-133b and miR-199b knockdown attenuate TGF- $\beta 1$-induced epithelial to mesenchymal transition and renal fibrosis by targeting SIRT1 in diabetic nephropathy. European Journal of Pharmacology 837: 96-104. DOI 10.1016/j.ejphar.2018.08.022.

Surowiak AK, Balcerzak L, Lochynski S, Strub DJ (2021). Biological activity of selected natural and synthetic terpenoid lactones.
International Journal of Molecular Sciences 22: 5036. DOI 10.3390/ijms22095036.

Shi Y, Huang J, Yi X, Yu S, Zhang L et al. (2017). MicroRNA-133b inhibits cell proliferation and invasion in osteosarcoma by targeting Sirt1. Oncology Research 25: 1421-1430. DOI 10.3727/096504016X14826089198805.

Tang BL (2016). Sirt1 and the mitochondria. Molecules and Cells 39: 87-95. DOI 10.14348/molcells.2016.2318.

Terman A, Brunk U (1998). On the degradability and exocytosis of ceroid/lipofuscin in cultured rat cardiac myocytes. Mechanisms of Ageing and Development 100: 145-156. DOI 10.1016/S0047-6374(97)00129-2.

Tian Z, Jiang H, Liu Y, Huang Y, Xiong X et al. (2016). MicroRNA133b inhibits hepatocellular carcinoma cell progression by targeting Sirt1. Experimental Cell Research 343: 135-147. DOI 10.1016/j.yexcr.2016.03.027.

Uckun FM, Saund S, Windlass H, Trieu V (2021). Repurposing antimalaria phytomedicine artemisinin as a COVID-19 drug. Frontiers in Pharmacology 12: 649532. DOI 10.3389/ fphar.2021.649532.

Vaziri H, Dessain SK, Ng Eaton E, Imai SI, Frye RA et al. (2001). hSIR2(SIRT1) functions as an NAD-dependent p53 deacetylase. Cell 107: 149-159. DOI 10.1016/S0092-8674 (01)00527-X.

Wang J, Zhai Y, Ou M, Bian Y, Tang C et al. (2021a). Protective effect of lemon peel extract on oxidative stress in $\mathrm{H} 9 \mathrm{c} 2$ rat heart cell injury. Drug Design Development and Therapy 15: 20472058. DOI 10.2147/DDDT.S304624.

Wang Z, Hao Y, Yu H, Wei P (2021b). Dihydroartemisinin prevents palmitate-induced $\beta$-cell apoptosis. Apoptosis 26: 147-149. DOI 10.1007/s10495-021-01660-6.

Wei Z, Jia J, Heng G, Xu H, Shan J et al. (2019). Sirtuin-1/ mitochondrial ribosomal protein S5 axis enhances the metabolic flexibility of liver cancer stem cells. Hepatology 70: 1197-1213. DOI 10.1002/hep.30622.

Wu B, Feng JY, Yu LM, Wang YC, Chen YQ et al. (2018). Icariin protects cardiomyocytes against ischaemia/reperfusion injury by attenuating sirtuin 1-dependent mitochondrial oxidative damage. British Journal of Pharmacology 175: 4137-4153. DOI 10.1111/bph.14457.

Xiong J, Dong X, Li S, Jiang F, Chen J et al. (2021). Effects of (Pro) renin receptor on diabetic cardiomyopathy pathological processes in rats via the PRR-AMPK-YAP pathway. Frontiers in Physiology 12: 657378. DOI 10.3389/ fphys.2021.657378.

Xu Y, Qu X, Zhou J, Lv G, Han D et al. (2021). Pilose antler peptide3.2KD ameliorates adriamycin-induced myocardial injury through TGF- $\beta / S M A D$ signaling pathway. Frontiers in Cardiovascular Medicine 8: 659643. DOI 10.3389/ fcvm.2021.659643.

Yoshioka H, Li A, Suzuki A, Ramakrishnan S, Zhao Z, Iwata J (2021). Identification of microRNAs and gene regulatory networks in cleft lip common in humans and mice. Human Molecular Genetics 30: 1881-1893. DOI 10.1093/hmg/ddab151.

Zang H, Wu W, Qi L, Tan W, Nagarkatti P et al. (2020). Autophagy inhibition enables Nrf2 to exaggerate the progression of diabetic cardiomyopathy in mice. Diabetes 69: 2720-2734. DOI $10.2337 / \mathrm{db} 19-1176$.

Zhang B, Li X, Liu G, Zhang C, Zhang X et al. (2021a). Peroxiredomin-4 ameliorates lipotoxicity-induced oxidative stress and apoptosis in diabetic cardiomyopathy. Biomedicine \& Pharmacotherapy 141: 111780. DOI 10.1016/j.biopha.2021.111780. 
Zhang Q, Deng Q, Zhang J, Ke J, Zhu Y et al. (2018). Activation of the Nrf2-ARE pathway ameliorates hyperglycemia-mediated mitochondrial dysfunction in podocytes partly through Sirt1. Cellular Physiology and Biochemistry 48: 1-15. DOI 10.1159/000491658.

Zhang Q, Yi H, Yao H, Lu L, He G et al. (2021b). Artemisinin derivatives inhibit non-small cell lung cancer cells through induction of ROS-dependent apoptosis/ferroptosis. Journal of Cancer 12: 4075-4085. DOI 10.7150/jca.57054.

Zhou Y, Huang H, Hou X (2019). MicroRNA-133b alleviates hypoxia injury by direct targeting on NOD-like receptor protein 3 in rat $\mathrm{H} 9 \mathrm{c} 2$ cardiomyocyte. Cardiology Research and Practice 2019: 1-8. DOI 10.1155/2019/ 8092461. 